\title{
Thermal Entanglement and Critical Behavior of Magnetic Properties on a Triangulated Kagomé Lattice
}

\author{
N. Ananikian, L. Ananikyan, L. Chakhmakhchyan, A. Kocharian
}

\begin{abstract}
The equilibrium magnetic and entanglement properties in a spin-1/2 Ising-Heisenberg model on a triangulated Kagomé lattice are analyzed by means of the effective field for the Gibbs-Bogoliubov inequality. The calculation is reduced to decoupled individual (clusters) trimers due to the separable character of the Ising-type exchange interactions between the Heisenberg trimers. The concurrence in terms of the three qubit isotropic Heisenberg model in the effective Ising field in the absence of a magnetic field is non-zero. The magnetic and entanglement properties exhibit common (plateau, peak) features driven by a magnetic field and (antiferromagnetic) exchange interaction. The (quantum) entangled and non-entangled phases can be exploited as a useful tool for signalling the quantum phase transitions and crossovers at finite temperatures. The critical temperature of order-disorder coincides with the threshold temperature of thermal entanglement.
\end{abstract}

Keywords: Triangulated Kagomé lattice, Ising-Heisenberg model, Gibbs-Bogoliubov inequality, entanglement, concurrence.

\section{Introduction}

Geometrically frustrated spin systems exhibit fascinating new phases of matter, a rich variety of unusual ground states and thermal properties as a result of zero and finite temperature phase transitions driven by quantum and thermal fluctuations, respectively [1]. The efforts aimed at a better understanding of these phenomena have stimulated an intensive search for transition-metal magnetic and molecular materials whose paramagnetic metal centers can be strongly frustrated by local geometric structures. One of the most interesting geometrically frustrated magnetic two-dimensional structures is the triangulated Kagomé (triangles-in-triangles) lattice, which can be applied to the magnet $\mathrm{Cu}_{9} \mathrm{X}_{2}(\mathrm{cpa})_{6} \cdot \mathrm{nH}_{2} \mathrm{O}$ (X $=\mathrm{F}, \mathrm{Cl}, \mathrm{Br}$ and cpa=carboxypentonic acid) [2]. The magnetic architecture of these series of compounds, which can be regarded as a triangulated Kagomé lattice (Fig. 1), is currently under active theoretical investigation [3]. The spin- $\frac{1}{2}$ IsingHeisenberg model on this lattice, which takes into account quantum interactions between $\mathrm{Cu}^{2+}$ ions in $a$-sites, in the limit when monomeric $b$-spins having an exchange of Ising character, provides a rich physics and displays the essential features of the copper based coordination compounds $[4,5]$.

Entanglement is a generic feature of quantum correlations in systems, which cannot be quantified classically [6]. It provides a new perspective for understanding quantum phase transitions (QPTs) and collective many-body phenomena in condensed matter physics. A key novel observation is that quantum entanglement can play an important role in proximity to QPTs controlled by quantum fluctuations in the vicinity of quantum critical points. A new line of research points to a connection between the entanglement of a many-particle system and the existence of QPTs and scaling [7]. The basic features of entanglement in spin- $\frac{1}{2}$ finite systems are fairly well understood by now, while the role of local cluster topology and spin correlations in the thermodynamic limit still remains unanswered. Effective field theories can be offered by using the Gibbs-Bogoliubov inequality for studying the thermodynamic and thermal entanglement properties of many-body systems [8]. Although the method is not exact, it is still possible to see regions of criticality [9].

Unlike a classical transition, controlled by temperature, a quantum phase transition (QPT) is driven solely by (quantum) interactions. In the case of the triangulated Kagomé lattice, each $a$ type trimer interacts with its neighboring trimer through the (ferromagnetic) Ising-type exchange, i.e. a classical interaction. Therefore, the states of two neighboring $a$-clusters become separable (unentangled) [6]. Thus, the concurrence (a measure of entanglement [10]), which characterizes quantum (non classical) features for each trimer, in the effective field, can be calculated separately. The key result of the current work is a comparative analysis of specific (peak and plateau) features in the magnetic and thermal entanglement properties of the spin- $1 / 2$ IsingHeisenberg model on a triangulated Kagomé lattice. 
The rest of the paper is organized as follows: in Sec. 2 we introduce the Ising-Heisenberg model on the triangulated Kagomé lattice and provide a variational solution based on the Gibbs-Bogoliubov inequality. The basic principles for calculating entanglement measure and some of the results on intrinsic properties are introduced in Sec. 3. In Sec. 4 we present a comparison of magnetic properties and thermal entanglement. Concluding remarks are given in Sec. 5.

\section{Basic formalism}

We consider the spin- $\frac{1}{2}$ Ising-Heisenberg model on a triangulated Kagomé lattice (TKL) (Fig. 1) consisting of two types of sites $(a$ and $b)$. Since the exchange coupling between $\mathrm{Cu}^{2+}$ ions is almost isotropic, it is more appropriate to apply isotropic Heisenberg model. There is a strong Heisenberg $J_{a a}$ (antiferro) exchange coupling between trimeric sites of type $a$ and a weaker Ising-type (ferro) exchange $\left(J_{a b}\right)$ between trimeric types $a$ and monomeric $b$. Thus, the Kagomé lattice of the Ising spins (monomers) contains inside each triangle unit a smaller triangle of Heisenberg spins (trimer).

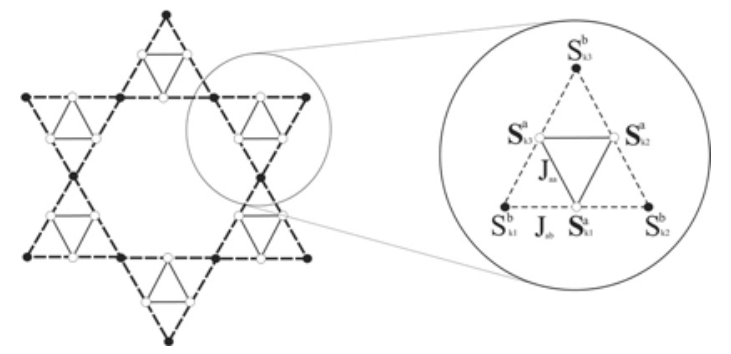

Fig. 1: A cross-section of TKL. The solid lines represent intra-trimer Heisenberg interactions $J_{a a}$, while the broken lines label monomer-trimer Ising interations $J_{a b}$. The circle marks the $k$-th cluster. $\mathbf{S}_{k_{i}}^{a}$ presents the Heisenberg spins and $S_{k_{i}}^{b}$ the Ising spins

The Hamiltonian can be written as follows:

$$
\begin{aligned}
\mathcal{H}= & J_{a a} \sum_{(i, j)} \mathbf{S}_{i}^{a} \mathbf{S}_{j}^{a}-J_{a b} \sum_{(k, l)}\left(S^{z}\right)_{k}^{a} \cdot\left(S^{z}\right)_{l}^{b}- \\
& H \sum_{i=1}^{\frac{2 N}{3}} 3\left[\left(S^{z}\right)_{j}^{a}+\frac{1}{2}\left(S^{z}\right)_{j}^{b}\right],
\end{aligned}
$$

where $\mathbf{S}^{a}=\left\{S_{x}^{a}, S_{y}^{a}, S_{z}^{a}\right\}$ is the Heisenberg spin- $\frac{1}{2}$ operator, and $S^{b}$ is the Ising spin. $J_{a a}>0$ corresponds to antiferro-coupling and $J_{a b}>0$ ferro-couplings. Here, the total number of sites is $3 N$, where the first two summations run over $a-a$ and $a-b$ nearest neighbors, respectively, and the last sum incorporates the effect of a uniform magnetic field.

The variational Gibbs-Bogoliubov inequality is adopted to solve the Hamiltonian (1)

$$
F \leq F_{0}+\left\langle\mathcal{H}-\mathcal{H}_{0}\right\rangle_{0},
$$

where $\mathcal{H}$ is the real Hamiltonian which describes the system, and $\mathcal{H}_{0}$ is the trial one. $F$ and $F_{0}$ are free energies corresponding to $\mathcal{H}$ and $\mathcal{H}_{0}$, respectively, and $\langle\ldots\rangle_{0}$ denotes the thermal average over the ensemble defined by $\mathcal{H}_{0}$. Following [4], the trial Hamiltonian is reduced to

$$
\begin{aligned}
\mathcal{H}_{0}= & \sum_{k \in \text { trimers }} \mathcal{H}_{c_{0}}, \\
\mathcal{H}_{c_{0}}= & \lambda_{a a}\left(\mathbf{S}_{k_{1}}^{a} \mathbf{S}_{k_{2}}^{a}+\mathbf{S}_{k_{2}}^{a} \mathbf{S}_{k_{3}}^{a}+\mathbf{S}_{k_{1}}^{a} \mathbf{S}_{k_{3}}^{a}\right)- \\
& \sum_{i=1}^{3}\left[\gamma_{a}\left(S^{z}\right)_{k_{i}}^{a}+\frac{\gamma_{b}}{2}\left(S^{z}\right)_{k_{i}}^{b}\right] .
\end{aligned}
$$

In this Hamiltonian, the stronger quantum Heisenberg antiferromagnetic interactions between $a$-sites are treated exactly, while the weaker Ising-type ones between the $a$ - and $b$-sites $\left(\left|J_{a b} / J_{a a}\right| \approx 0.025[11]\right)$ are replaced by self-consistent (effective) fields of two types: $\gamma_{a}$ and $\gamma_{b}$.

The variational parameters $\gamma_{a}, \gamma_{b}$ and $\lambda_{a a}$ can be found by minimizing the RHS of (2). Using the fact that in terms of $(3 \mathrm{~b}) \mathbf{S}^{a}$ and $\mathbf{S}^{b}$ are statistically independent, and taking into account $\left\langle S^{x}\right\rangle_{0}=$ $\left\langle S^{y}\right\rangle_{0}=0$ a single site magnetization, $\left\langle\left(S^{z}\right)^{a}\right\rangle_{0}=m_{a}$, $\left\langle\left(S^{z}\right)^{b}\right\rangle_{0}=m_{b}$ on $a$ and $b$-sites, we obtain $\lambda_{a a}=J_{a a}$, $\gamma_{a}=2 J_{a b} m_{b}+H, \gamma_{b}=4 J_{a b} m_{a}+H$. The eigenvalues of $\mathcal{H}_{c_{0}}^{a}$ are:

$$
\begin{aligned}
& E_{1}=\frac{3}{4}\left(\lambda_{a a}+2 \gamma_{a}\right) ; E_{2}=E_{3}=\frac{1}{4}\left(-3 \lambda_{a a}+2 \gamma_{a}\right) \\
& E_{4}=\frac{1}{4}\left(3 \lambda_{a a}+2 \gamma_{a}\right) ; E_{5}=E_{6}=\frac{1}{4}\left(-3 \lambda_{a a}-2 \gamma_{a}\right) \\
& E_{7}=\frac{1}{4}\left(3 \lambda_{a a}-2 \gamma_{a}\right) ; E_{8}=\frac{3}{4}\left(\lambda_{a a}-2 \gamma_{a}\right)
\end{aligned}
$$

and the corresponding eigenvectors given by

$$
\begin{aligned}
\left|\psi_{1}\right\rangle & =|000\rangle \\
\left|\psi_{2}\right\rangle & =\frac{1}{\sqrt{3}}\left(q|001\rangle+q^{2}|010\rangle+|100\rangle\right) \\
\left|\psi_{3}\right\rangle & =\frac{1}{\sqrt{3}}\left(q^{2}|001\rangle+q|010\rangle+|100\rangle\right) \\
\left|\psi_{4}\right\rangle & =\frac{1}{\sqrt{3}}(|001\rangle+|010\rangle+|100\rangle) \\
\left|\psi_{5}\right\rangle & =\frac{1}{\sqrt{3}}\left(q|110\rangle+q^{2}|101\rangle+|011\rangle\right) \\
\left|\psi_{6}\right\rangle & =\frac{1}{\sqrt{3}}\left(q^{2}|110\rangle+q|101\rangle+|011\rangle\right) \\
\left|\psi_{7}\right\rangle & =\frac{1}{\sqrt{3}}(|110\rangle+|101\rangle+|011\rangle) \\
\left|\psi_{8}\right\rangle & =|111\rangle,
\end{aligned}
$$

where $q=e^{i 2 \pi / 3}$ (these eigenvectors should be also the eigenstates of cyclic (rotation) operator $P$ with eigenvalues $1, q$ and $q^{2}$, satisfying $\left.q^{2}+q+1=0\right)$. 
For the above-defined $a$ - and $b$-single site magnetizations we obtain (here and further the Boltzman's constant is set to be $\left.k_{B}=1\right)$ :

$$
\begin{aligned}
& m_{a}= \\
& \frac{1}{6} \frac{3 \sinh \left(\frac{3 \gamma_{a}}{2 T}\right)+2 e^{\frac{3 \lambda_{a a}}{2 T}} \sinh \left(\frac{\gamma_{a}}{2 T}\right)+\sinh \left(\frac{\gamma_{a}}{2 T}\right)}{\cosh \left(\frac{3 \gamma_{a}}{2 T}\right)+2 e^{\frac{3 \lambda_{a a}}{2 T}} \cosh \left(\frac{\gamma_{a}}{2 T}\right)+\cosh \left(\frac{\gamma_{a}}{2 T}\right)}, \\
& m_{b}=\frac{1}{2} \tanh \left(\frac{\gamma_{b}}{2 T}\right) .
\end{aligned}
$$

For the Gibbs-Bogoliubov free energy $\left(F_{G B}\right)$ of the system we obtain the following expression:

$$
\begin{aligned}
& \frac{F_{G B}}{N}=\frac{\lambda_{a a}}{2}+4 J_{\mathrm{ab}} m_{a} m_{b}-2 T\left[\frac { 1 } { 3 } \operatorname { l n } \left\{4 e^{\frac{3 J_{\mathrm{ab}}}{2 T}}\right.\right. \\
& \left.\cosh \left(\frac{\gamma_{a}}{2 T}\right)+2 \cosh \left(\frac{\gamma_{a}}{2 T}\right)+2 \cosh \left(\frac{3 \gamma_{a}}{2 T}\right)\right\}+ \\
& \left.\frac{1}{2} \ln \left\{2 \cosh \left(\frac{\gamma_{b}}{2 T}\right)\right\}\right] .
\end{aligned}
$$

\section{Concurrence and thermal entanglement}

The effective field treatment of (1) transforms manybody system to a reduced "single" cluster study. This allows us to study, in particular, the thermal (local) entanglement properties of the $a$-sublattice in terms of a three-qubit (isotropic) Heisenberg model in a self-consistent field $\gamma_{a}$, which carries the properties of the whole system. As a measure of the pairwise entanglement, we use concurrence $C(\rho)[10]$. The corresponding density matrix $\rho$ is defined as

$$
C(\rho)=\max \left\{\lambda_{1}-\lambda_{2}-\lambda_{3}-\lambda_{4}, 0\right\},
$$

where $\lambda_{i}$ are the square roots of the eigenvalues of the operator $\tilde{\rho}=\rho_{12}\left(\sigma_{1}^{y} \otimes \sigma_{2}^{y}\right) \rho_{12}^{*}\left(\sigma_{1}^{y} \otimes \sigma_{2}^{y}\right)$ in descending order. Since we consider pairwise entanglement, we use the reduced density matrix $\rho_{12}=\operatorname{Tr}_{3} \rho$. In the effective field, due to the classical character of the Ising interaction (Sec. 1) between trimers, the concurrence for each decoupled Heisenberg cluster can be calculated individually. In our case, the density matrix has the form $\rho=\frac{1}{Z} \sum_{k=1}^{8} \exp \left(-E_{k} / T\right)\left|\psi_{k}\right\rangle$ $\left\langle\psi_{k}\right|, E_{k}$ and $\left|\psi_{k}\right\rangle$ taken from (4) and (5) and $Z$ is the partition function $[Z=\operatorname{Tr} \rho=$ $\left.e^{-\frac{3\left(2 \gamma_{a}+\lambda_{a a}\right)}{4 T}}\left(1+e^{\frac{\gamma_{a}}{T}}\right)\left(1+e^{\frac{2 \gamma_{a}}{T}}+2 e^{\frac{2 \gamma_{a}+3 \lambda_{a a}}{2 T}}\right)\right]$.

While the construction of $\tilde{\rho}$ does not depend on whether $\gamma_{a}$ is an effective parameter or a real magnetic field, the self-consistent field solution for $\gamma_{a}$ is crucial in obtaining the final results. In this paper we skip specific derivations and rather focus only on the final results. Concurrence $C(\rho)$ is given by [12]:

$$
C(\rho)=\frac{2}{Z} \max (|y|-\sqrt{u v}, 0)
$$

where

$$
\begin{aligned}
& u=\frac{1}{3} e^{\frac{2 \gamma_{a}-3 \lambda_{\mathrm{aa}}}{4 T}}\left(1+3 e^{\frac{\gamma_{a}}{T}}+2 e^{\frac{3 \lambda_{\mathrm{aa}}}{2 T}}\right) \\
& v=\frac{1}{3} e^{-\frac{3\left(2 \gamma_{a}+\lambda_{\mathrm{aa}}\right)}{4 T}}\left(3+e^{\frac{\gamma_{a}}{T}}+2 e^{\frac{2 \gamma_{a}+3 \lambda_{\mathrm{aa}}}{2 T}}\right) \\
& w=\frac{1}{3} e^{-\frac{2 \gamma_{a}+3 \lambda_{\mathrm{aa}}}{4 T}}\left(1+e^{\frac{\gamma_{a}}{T}}\right)\left(1+2 e^{\frac{3 \lambda_{\mathrm{aa}}}{2 T}}\right) \\
& y=-\frac{1}{3} e^{-\frac{2 \gamma_{a}+3 \lambda_{\mathrm{aa}}}{4 T}}\left(1+e^{\frac{\gamma_{a}}{T}}\right)\left(-1+e^{\frac{3 \lambda_{\mathrm{aa}}}{2 T}}\right) .
\end{aligned}
$$

First, we find that concurrence $C(\rho)$ as an entanglement measure exhibits critical behavior upon the temperature variation shown in Fig. 2 in the absence of a field.

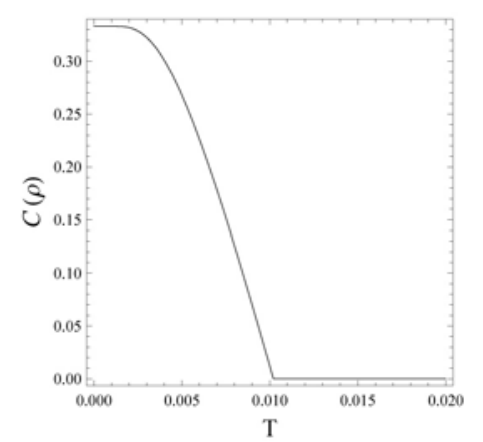

Fig. 2: Concurrence $C(\rho)$ versus temperature field for $J_{a a}=1, \alpha=0.025$ and $H=0$

The system is entangled at a relatively low temperature, below the threshold, $T_{t h}$. This effect apparently occurs because of the Ising-type interaction replaced by the effective field $\gamma_{a}=2 J_{a b} m_{b}+H$ acting upon the $a$-spins, which isnon-zero at $H=0$. Thus, effective field provides a solution for an entanglement resource in the absence of a magnetic field.

Another important observation: the threshold temperature at which $C(\rho)$ becomes zero coincides with the critical temperature $T_{c}$ at which spontaneous magnetization $m$ vanishes for the smooth (second order) phase transition between ordereddisordered phases. Expanding $m_{a}$ into series near the phase transition point:

$$
m=a m+b m^{3}+c m^{5}+\ldots
$$

one finds $T_{c}$ from the condition $a=1, b<0$ (for the case $J_{a a}=1$ and $\alpha=0.025, T_{c}=0.0102062$ ). The coincidence of the critical and threshold temperatures for magnetization and concurrence is a consequence of the fact that at $T_{c}$ the system undergoes the order-disorder phase transition and the second term in $\gamma_{a}$ also vanishes $\left(m_{b}=0\right.$, when $H=0$ and $T \geq T_{c}$ ). In general, we find a number of other similarities between the magnetic properties and the entanglement of the system. Under variation of $H$, the entanglement and magnetic properties show very 
rich behavior in the low-temperature region. Fig. 3 presents the three-dimensional plot of the concurrence as a function of the temperature and external magnetic field.

We will study some other features in the behavior of $C(\rho)$ by returning to the magnetic and entanglement ground state properties in Sec. 4 .

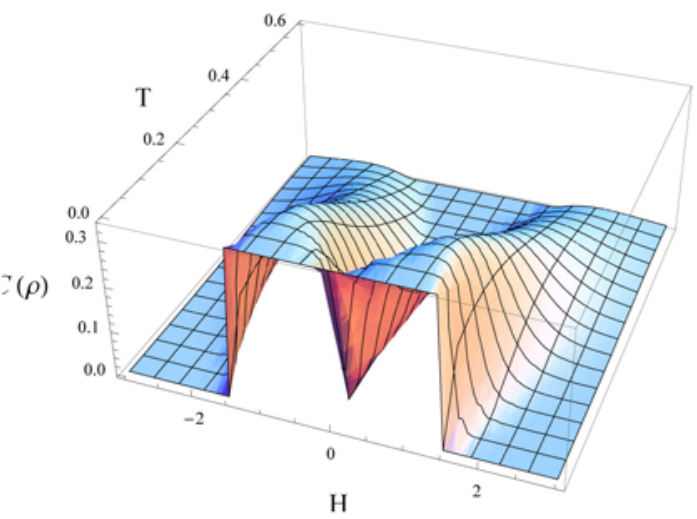

Fig. 3: Concurrence $C(\rho)$ versus temperature $T$ and external magnetic field $H$ for $J_{a a}=1, \alpha=0.025$

\section{Quantum critical points and phase diagrams}

Now we consider the many-body quantum effects relevant to entanglement properties and discuss some similarities between magnetic (statistical) properties and (quantum) entanglement. As statistical characteristics, the density distribution of susceptibility $\chi=\frac{\partial m_{a}}{\partial H}$ reduced per one $a$-site is shown in Fig. 4 (a) as a function of the coupling constant $J_{a a}$ and the external field $H$, at a relatively high temperature $T=0.1$, higher than $T_{c}$. The white stripes, which have a certain a finite width due to nonzero temperature, correspond to the peaks of the susceptibility. A similar density plot is shown for entanglement in Fig. 4 (b) for the same range of $J_{a a}-H$ parameters. A comparison of these two graphs shows that the general behavior of the statistical properties in $\chi$ resembles the features of the quantum concurrence. (a)

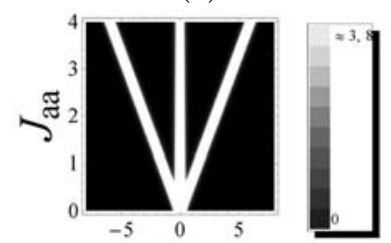

$\mathrm{H}$ (b)

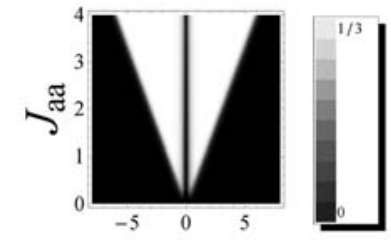

$\mathrm{H}$
Fig. 4: Density plot of (a) susceptibility $\chi$, (b) concurrence $C(\rho)$ versus $H$ and $J_{a a}$ for $\alpha=0.025$ and $T=0.1$
Our calculations show that the peaks in magnetic susceptibility correspond to the phase boundaries on the $J_{a a}-H$ density diagram in concurrence $C(\rho)$ at which the quantum coherence vanishes. As can be seen, this is true only for the Ising-Heisenberg model on the TKL lattice with $J_{a a}>0$ coupling; for $J_{a a}<0$ coupling the system is non-entangled, $C(\rho)=0$.

Thus, the extremal behavior of $\chi$ is not reproduced by the concurrence density. Hence, although the critical behavior of the two characteristics coincides for the antiferromagnetic region, only (quantum) concurrence can be used as a reference for quantitative analysis of QPTs.

In addition, we study here the quantum criticality in the ground state phase diagram resulting from the magnetic field variation in the magnetization and entanglement properties of the $a$-sublattice. Fig. 5(a) shows a phase diagram of constant magnetization for the $a$-sublattice. This diagram differentiates the following phases for $J_{a a}>0$ : Phase $\mathbf{I}$ corresponds to the spontaneous magnetization $m_{a}=1 / 6$, when spins in the $a$-sublattice are in one of the available $(\uparrow \uparrow \downarrow)$ configurations; Phase II corresponds to one of the possible configurations $(\downarrow \downarrow \uparrow)$ with $m_{a}=-1 / 6$. (a)

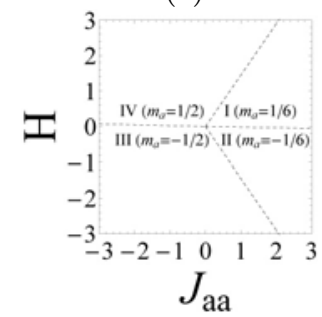

(b)

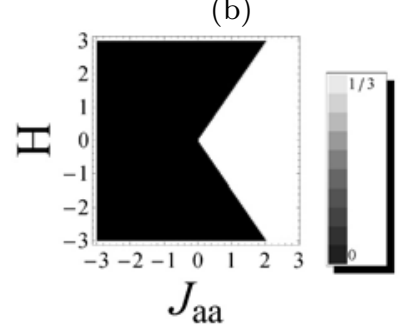

Fig. 5: (a) Phase diagram of the $a$-sublattice for $|\alpha|=$ 0.025 ; (b) Density plot of concurrence $C(\rho)$ versus $H$ and $J_{a a}$ for $|\alpha|=0.025$ at zero temperature

In the ferromagnetic case $\left(J_{a a}<0\right)$ we have full spin saturation in regions III and IV, with the value of the maximum magnetization per atom $m_{a}=1 / 2$ [configuration $(\downarrow \downarrow \downarrow)]$ and $m_{a}=-1 / 2$ [configuration $(\uparrow \uparrow \uparrow)]$, respectively. Phase $\mathbf{I}$ contains the two-fold degenerate states $\left|\psi_{5}\right\rangle$ and $\left|\psi_{6}\right\rangle$, while Phase II contains the two-fold degenerate states $\left|\psi_{2}\right\rangle$ and $\left|\psi_{3}\right\rangle$ with $C(\rho)=1 / 3$. Phases III and IV correspond to states $\left|\psi_{1}\right\rangle$ and $\left|\psi_{8}\right\rangle$, respectively. These phases are non-entangled, $(C(\rho)=0)$ [Fig. 5(b)]. The area of non-zero entanglement coincides with phase $\mathbf{I}+\mathbf{I I}$, where $\left|m_{a}\right|=1 / 6$, while the one with zero entanglement $(C(\rho)=0)$ corresponds to phase III + IV with $\left|m_{a}\right|=1 / 2$.

\section{Conclusion}

In this work we have demonstrated correlations between magnetic properties and quantum entanglement in the spin- $\frac{1}{2}$ Ising-Heisenberg model on a tri- 
angulated Kagomé lattice. We adopted the variational mean-field like treatment (based on the GibbsBogoliubov inequality) to decouple clusters in effective (interconnected) fields of two types (consisting of Heisenberg $a$ trimers and Ising-type $b$ monomers). Each of these fields taken separately describes not only the corresponding ( $a$ - or $b$ - type) spins, but the system as a whole.

We used concurrence as a computable measure of bipartite entanglement for the trimeric units in terms of the isotropic Heisenberg model in the effective magnetic field $\gamma_{a}$. Using the fact that " $a$ subdivisions" are separable, we studied the entanglement for each of them individually in an effective Isingtype field, $\left(\gamma_{b}\right)$. The model exhibits quantum criticality, which can be identified and characterized by studying the behavior of the magnetic and entanglement properties with respect to the interaction, the magnetic field and the temperature that control the transition. It turned out that entanglement does not vanish in the zero external field, as happens for the common three qubit (isotropic) Heisenberg model.

We find that the temperature at which entanglement becomes zero coincides with the critical temperature of the second order phase transition at which spontaneous magnetization disappears. In addition, we show that in the antiferromagnetic region (the interactions between trimeric $a$ sites are exactly of this type) the magnetic susceptibility peaks coincide with the boundary lines at which entanglement vanishes. However, this does not take place in the ferromagnetic case. Therefore, one can detect a quite visible correlation for the line boundaries between the phases on the density diagrams for entanglement and magnetization as a signature of the corresponding quantum phase transition. Note that the disordered spin liquid state can also exist in the ground state of the frustrated spin system, on the assumption that there is a sufficiently strong antiferromagnetic intra-trimer interaction.

Finally, the magnetization, magnetic susceptibilities and (quantum) entanglement features can be exploited to signal and understand the quantum critical points and phase transitions.

\section{Acknowledgement}

This work was supported by the PS-1981, PS2033 ANSEF and ECSP-09-08-sasp NFSAT research grants.

\section{References}

[1] Diep, H. T. (ed.): Frustrated Spin Sysytems. Singapore : World Scientific, 2004; Gardner, J. S., et al.: Magnetic Pyrochlore Oxides. Rev. Mod. Phys., 82(1), 2010, p. 53-107; Zhito- mirsky, M. E., et al.: Field Induced Ordering in Highly Frustrated Antiferromagnets. Phys. Rev. Lett., 85(15), 2000, p. 3269-3272.

[2] Maruti, S., Haar ter, L. W.: Magnetic Properties of the Two-Dimensional Triangles-in-Triangles Kagomé Lattice $\mathrm{Cu}_{9} \mathrm{X}_{2}(\mathrm{cpa})_{6} \quad(\mathrm{X}=\mathrm{F}, \mathrm{Cl}, \mathrm{Br})$. J. Appl. Phys., 75(10), 1994, p. 5949-5952; Ateca, S., et al.: The Two-Dimensional Triangles-in-Triangles Kagomé Antiferromagnet $\mathrm{Cu}_{9} \mathrm{X}_{2}(\mathrm{cpa})_{6}$ : a Topological Spin-Glass? J. Magn. Magn. Mater., 147(3), 1995,

p. 398-400; Ramirez, A. P.: Strongly Geometrically Frustrated Magnets. Annu. Rev. Mater. Sci., 24, 1994, p. 453-480.

[3] Strečka, J., et al.: Exact Solution of the Geometrically Frustrated Spin-1/2 Ising-Heisenberg Model on the Triangulated Kagome (Trianglesin-Triangles) Lattice. Phys. Rev. B, 78(2), 2008, 024427; Ryo, N., et al.: A Theoretical Study of Magnons for Heisenberg Model in Triangulated Kagomé Lattice-Characteristic Dispersionless Modes. J. Phys. Soc. Jpn., 66, 1997, p. $3687-3688$.

[4] Strečka, J.: Magnetic Properties of the Geometrically Frustrated Spin-1/2 Heisenberg Model on the Triangulated Kagomé Lattice. J. Magn. Magn. Mater., 316(2), 2007, p. e346-e348.

[5] Yao, D.-X., et al.: XXZ and Ising Spins on the Triangular Kagome Lattice. Phys. Rev. B, 78(2), 2008, 024428.

[6] Amico, L., et al.: Entanglement in ManyBody Systems. Rev. Mod. Phys., 80(2), 2008, p. 517-576; Amico, L., Fazio, R.: Entanglement and Magnetic Order. J. Phys. A: Math. Theor., 42(50), 2009, 504001.

[7] Larsson, D., Johannesson, H.: Entanglement Scaling in the One-Dimensional Hubbard Model at Criticality. Phys. Rev. Lett., 95(19), 2005, 196406; Yang, C., et al.: Phase Transitions and Exact Ground-State Properties of the OneDimensional Hubbard Model in a Magnetic Field. J. Phys.: Condens. Matter, 12(33), 7433; Alba, V., et al.: Entanglement Entropy of Two Disjoint Blocks in Critical Ising Models. Phys. Rev. B, 81(6), 2010, 060411(R).

[8] Gong, S.-S., Gang, S.: Thermal Entanglement in One-Dimensional Heisenberg Quantum Spin Chains under Magnetic Fields. Phys. Rev. A, 80(1), 2009, 012323; Asoudeh, M., Karimipour, V.: Thermal Entanglement of Spins in Mean-Field Clusters. Phys. Rev. A 73(6), 2006, 062109; Canosa, N., et al.: Description of Thermal Entanglement with the Static Path Plus 
Random-Phase Approximation. Phys. Rev. A, 76(2), 2007, 022310 .

[9] Vedral, V.: Mean-Field Approximations and Multipartite Thermal Correlations. New J. Phys., 6(1), 2004, 22.

[10] Wootters, W. K.: Entanglement of Formation of an Arbitrary State of Two Qubits. Phys. Rev. Lett., 80(10), 1998, p. 2245-2248.

[11] Mekata, M., al.: Magnetic Ordering in Triangulated Kagomé Lattice Compound

$\mathrm{Cu}_{9} \mathrm{Cl}_{2}(\mathrm{cpa})_{6} \mathrm{nH}_{2} \mathrm{O}$. J. Magn. Magn. Mater., 177-181, 1998, p. 731-732.

[12] Wang, X., et al.: Thermal Entanglement in Three-Qubit Heisenberg Models. J. Phys. A: Math. Gen., 34(50), 2001, 11307.
N. Ananikian

Yerevan Physics Institute

Alikhanian Br. 2

0036 Yerevan, Armenia

L. Ananikyan

E-mail: lev.ananikyan@gmail.com

Yerevan Physics Institute

Alikhanian Br. 2

0036 Yerevan, Armenia

L. Chakhmakhchyan

Yerevan Physics Institute

Alikhanian Br. 2

0036 Yerevan, Armenia

Yerevan State University

A. Manoogian 1

0025 Yerevan, Armenia

A. Kocharian

Yerevan Physics Institute

Alikhanian Br. 2

0036 Yerevan, Armenia

Department of Physics

California State University

Los Angeles, CA 90032, USA 